\title{
Three-Dimensional Imaging of Nanoparticle Chemistry Using Spectroscopic Single Particle Reconstruction
}

Yi-Chi Wang ${ }^{1}$, Thomas J A Slater ${ }^{1}{ }^{2 *}$, Gerard M Leteba ${ }^{3}$, Alan M Roseman ${ }^{4}$, Christopher P Race ${ }^{1}$, Neil P Young 5 , Angus I Kirkland ${ }^{2,5}$, Candace I Lang ${ }^{6}$ and Sarah J Haigh ${ }^{1 *}$

1. School of Materials, University of Manchester, Oxford Road, Manchester, M13 9PL, U.K.

2. Electron Physical Sciences Imaging Centre, Diamond Light Source Ltd., Oxfordshire OX11 0DE, United Kingdom

3. Catalysis Institute, Department of Chemical Engineering, University of Cape Town, Rondebosch 7701, South Africa

4. Division of Molecular and Cellular Function, School of Biological Sciences, Faculty of Biology, Medicine and Health, Manchester Academic Health Science Centre, University of Manchester, Manchester M13 9PL, United Kingdom

5. Department of Materials, University of Oxford, Parks Road, Oxford OX1 3PH, United Kingdom

6. School of Engineering, Macquarie University, Macquarie Park, NSW 2109, Australia

* Corresponding authors: Sarah.haigh@manchester.ac.uk, Thomas.slater@diamond.ac.uk

Applications of nanoparticles have been found in many fields, from catalysis for the reduction of toxic automobile exhaust emissions to medical imaging and drug delivery [1]. Multi-element nanoparticles such as platinum-nickel (PtNi) have improved activity and reduced cost of production in comparison to their single-element counterparts [2]. Understanding the local chemical distribution in these nanoparticles is critically important to control their properties and performance. Scanning transmission electron microscope (STEM) imaging combined with energy dispersive X-ray spectroscopy (EDS) [3] or electron energy loss spectroscopy (EELS) [4] are two of the few techniques able to characterize local chemistry in inorganic nanoparticles. For complex chemical distributions, three-dimensional (3D) imaging provides a direct visualization of the specimen in its native $3 \mathrm{D}$ form and prevents complicated interpretation using only 2D projections. However, a conventional tilt-series electron tomography (ET) acquisition scheme imparts a high electron dose that may damage nanoparticles during acquisition. Therefore, we demonstrate a new 3D chemical imaging approach [5], named spectroscopic single particle reconstruction, built on the cryo-TEM single particle reconstruction (SPR) method that awarded the Nobel Prize in Chemistry in 2017 [6]. We apply this technique to the imaging of PtNi nanocatalysts and find complex elemental segregation within these alloyed nanoparticles.

STEM high-angle annular dark field (STEM-HAADF) and STEM-EDS images were simultaneously acquired for over one thousand $\mathrm{PtNi}$ nanoparticles. Inorganic nanoparticles are typically less homogenous in size and shape than proteins and viruses, so selection criteria were applied to fulfil the assumption of the SPR requirements, that all objects imaged are identical and are randomly orientated on a support. Figure 1 shows an example 2D HAADF image and the associated EDS maps containing tens of similar nanoparticles at different orientations. After image segmentation and selection [7], all individual nanoparticles with unknown orientations serve as the SPR input dataset. To solve the unknown orientations of each particle imaged, we performed a separate conventional STEM-HAADF tilt series ET reconstruction for a single PtNi particle. The 3D ET reconstruction serves as an initial morphological estimate that can be used to produce STEM-HAADF re-projections with known orientations. Orientations can then be assigned to each nanoparticle in the SPR data by cross correlating the ET STEM-HAADF re-projections with the SPR STEM-HAADF experimental images. Once the 
orientations are known it is then possible to reconstruct 3D HAADF and EDS intensities. Furthermore, we applied 3D EDS quantification to illustrate the complex chemical segregation in these nanoparticles (Figure 2).

In conclusion, our spectroscopic single particle reconstruction approach allowed 3D elemental mapping at nanometer resolution with an electron fluence per particle that is 500 times lower than would be required to achieve the same results using conventional STEM-EDS ET techniques. We find new evidence of a complex inhomogeneous alloying in PtNi nanocatalysts with a Pt-rich core, a Ni-rich hollow octahedral intermediate shell and a Pt-rich rhombic dodecahedral skeleton framework with less $\mathrm{Pt}$ at $\langle 100\rangle$ vertices. The approach we developed can also be extended to other spectroscopic signals available in the microscope. For example, EELS could be used to map elemental distributions or oxidation states of geometric nanoparticles using the technique [8].

\section{References:}

[1] P Strasser et al., Chem. Soc. Rev. 47 (2018), p. 715.

[2] K D Gilroy et al., Chem. Rev. 116 (2016), p. 10414.

[3] T J A Slater et al., Ultramicroscopy 162 (2016), p. 61.

[4] G Haberfehlner et al., Nanoscale 6 (2014), p. 14563.

[5] Y C Wang et al., Nano Letters 19 (2019), p. 732.

[6] Nobelprize.org. The Nobel Prize in Chemistry 2017 [Online].

http://www.nobelprize.org/nobel_prizes/chemistry/laureates/2017/ (accessed Apr 10, 2018)

[7] Y C Wang et al., Journal of Physics: Conference Series (2017), p. 012018.

[8] The authors acknowledge funding from the University of Manchester, European Research Council grant EvoluTEM, EPSRC grants (EP/G035954/1, EP/K016946/1, EP/J021172/1 and EP/P009050/1), NowNANO CDT, Defense Threat Reduction Agency Grant (HDTRA1-12-1-0013) and China Scholarship Council.
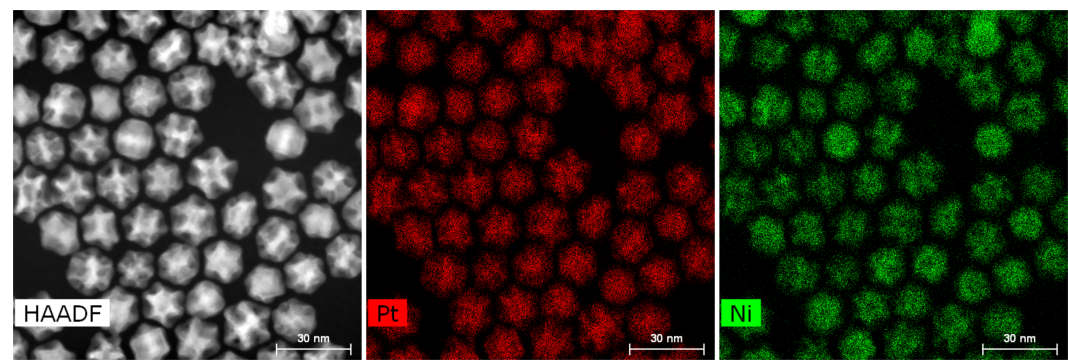

Figure 1. Example of PtNi nanoparticle morphology and elemental distribution in 2D projections. (a), HAADF image, (b) EDS Pt spectrum image and (c) EDS Ni spectrum image.

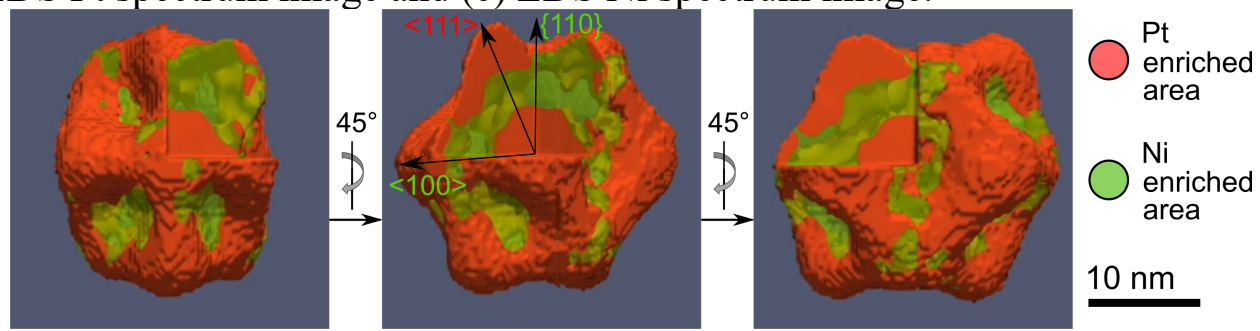

Figure 2. Three-dimensional visualization of reconstructed elemental segregation in PtNi nanoparticle population after 3D EDS quantification. 